\title{
Switchgrass Rhizosphere Metabolite Chemistry Driven by Nitrogen Availability
}

\author{
Darian N. Smercina, ${ }^{1, \dagger}$ Alan W. Bowsher, ${ }^{2}$ Sarah E. Evans, ${ }^{3}$ Maren L. Friesen, ${ }^{4}$ Elizabeth K. Eder, ${ }^{5}$ David W. Hoyt, ${ }^{5}$ \\ and Lisa K. Tiemann ${ }^{1}$ \\ ${ }^{1}$ Department of Plant, Soil and Microbial Sciences, Michigan State University, East Lansing, MI \\ ${ }^{2}$ Plant Resilience Institute, Michigan State University, East Lansing, MI \\ ${ }^{3}$ W.K. Kellogg Biological Station, Department of Integrative Biology, Michigan State University, Hickory Corners, MI \\ ${ }^{4}$ Department of Plant Pathology and Department of Crop and Soil Sciences, Washington State University, Pullman, WA \\ ${ }^{5}$ Environmental Molecular Sciences Laboratory, Pacific Northwest National Laboratory, Richland, WA
}

Accepted for publication 1 March 2020.

\section{ABSTRACT}

Plants and soil microorganisms interact closely in the rhizosphere where plants may exchange carbon $(\mathrm{C})$ for functional benefits from the microbial community. For example, the bioenergy crop, switchgrass (Panicum virgatum) is thought to exchange root-exuded $\mathrm{C}$ for nitrogen (N) fixed by diazotrophs (free-living $\mathrm{N}$-fixers). However, this interaction is not well characterized and it is not known how or if switchgrass responds to diazotrophs or their activity. To explore this question, we assessed rhizosphere metabolite chemistry of switchgrass grown in a hydroponic system under two $\mathrm{N}$ levels and under inoculated or uninoculated conditions. Plants were grown with the inoculum Azotobacter vinelandii DJ for 3 days before harvest. We found switchgrass root exudate chemistry to be driven by N availability. Total metabolite concentrations were generally greater under high $\mathrm{N}$ versus low $\mathrm{N}$ and unaffected by inoculation. Examination of rhizosphere chemical fingerprints indicates metabolite chemistry was also driven strongly by $\mathrm{N}$ availability with a greater relative abundance of carbohydrates under high $\mathrm{N}$ and greater relative abundance of organic acids under low $\mathrm{N}$. We also found evidence of changes in rhizosphere chemical fingerprints by inoculation treatment. However, we found little evidence of $\mathrm{N}$ treatment and inoculation interaction effects that suggests this response is not directly mediated by $\mathrm{N}$ availability.

Keywords: metabolomics, nitrogen fixation, plant-microbe interactions, rhizosphere, root exudates, soil ecology, switchgrass

\section{${ }^{\dagger}$ Corresponding author: D. Smercina; marinisd@msu.edu}

Funding: This work was conducted under the MMPRNT project, funded by the Department of Energy (DOE) Biological and Environmental Research (BER) Office of Science award DE-SC0014108. This work was also funded in part by the DOE Great Lakes Bioenergy Research Center (DOE BER Office of Science DE-SC0018409 and DE-FC02-07ER64494), by the National Science Foundation Long-Term Ecological Research Program (DEB 1832042) at the Kellogg Biological Station, and by Michigan State University AgBioResearch. A portion of the research was performed under the Facilities Integrating Collaborations for User Science (FICUS) program and used resources at the DOE Joint Genome Institute (JGI) and the Environmental Molecular Sciences Laboratory (EMSL; grid.436923.9), which are DOE Office of Science user facilities. Both facilities are sponsored by the Office of Biological and Environmental Research and operated under contract numbers DE-AC02-05CH11231 (JGI) and DEAC05-76RL01830 (EMSL). In particular, the metabolomics profiling measurements were made using EMSL instrumentation (Proposal ID 49977).

*The $e$-Xtra logo stands for "electronic extra" and indicates that supplementary material and three supplementary tables are published online.

The author(s) declare no conflict of interest.
Plants and soil microorganisms interact closely in the rhizosphere, where roots exude a variety of compounds including carbohydrates, organic acids, and amino acids, which influence the rhizosphere environment and the microbes that live there (Bais et al. 2006; Bertin et al. 2003; Jones et al. 2009). Through these interactions, plants may exchange carbon (C) for functional benefits conferred by the soil microbial community, such as protection against pathogens or mobilization of soil nutrients (Bais et al. 2006; Jones et al. 2009). Root exudation is thought to be mainly driven by passive diffusion (Jones et al. 2009), yet more active routes such as anion channels and ATP-driven transport highlight the potential for plants to regulate both the amount and composition of exudates released (Bertin et al. 2003; Sasse et al. 2018). Through "modulation of their exudate profiles" (i.e., changes in chemical composition and abundance), plants could alter their rhizobiome (Sasse et al. 2018). Yet, it is still unclear if plants alter root exudate chemistry in response to the rhizosphere community's functional capacity, such as the ability of the rhizobiome to fix nitrogen $(\mathrm{N})$. We explored this question using the interaction between switchgrass (Panicum virgatum) and the known diazotroph (free-living N-fixing organism) Azotobacter vinelandii DJ. 
Switchgrass, a perennial C4 grass native to North America, is a promising cellulosic bioenergy crop favored for its high biomass production and climate change mitigation potential (Robertson et al. 2017). Switchgrass productivity is often unresponsive to fertilizer $\mathrm{N}$ additions, resulting in similar biomass yields with fertilizer $\mathrm{N}$ additions well above or below plant $\mathrm{N}$ demands (Parrish and Fike 2005; Ruan et al. 2016). Further, yields remain consistently high despite $\mathrm{N}$ removal via yearly harvest (Parrish and Fike 2005; Ruan et al. 2016). An $\mathrm{N}$ mass balance suggests that switchgrass is accessing an unaccounted-for $\mathrm{N}$ source at rates of 35 to $58 \mathrm{~kg}$ of N ha ${ }^{-1}$ year $^{-1}$ (Roley et al. 2018). Switchgrass is also known to grow productively on low fertility marginal lands, which are often low in N (Robertson et al. 2017). This raises the question of how switchgrass is meeting its $\mathrm{N}$ demands.

One potential source of $\mathrm{N}$ for switchgrass is free-living $\mathrm{N}$-fixation (FLNF) carried out by diazotrophs in the rhizosphere. There is strong evidence for this as FLNF has been measured in association with the switchgrass rhizosphere (Roley et al. 2018, 2019; Smercina et al. 2019b). In fact, Roley et al. (2018) found that FLNF could account for 80 to $100 \%$ of the estimated "missing" N. Switchgrass is also known to host a diverse community of diazotrophs in its rhizosphere (Bahulikar et al. 2014). Switchgrass-diazotroph interactions present a unique system for exploring plant response to rhizobiome functional capacity given their known association and high potential for switchgrass to benefit from FLNF carried out by diazotrophs.

In this study, we explored the interaction between switchgrass and $A$. vinelandii DJ under high and low $\mathrm{N}$ conditions. High $\mathrm{N}$ should be less favorable than low $\mathrm{N}$ for FLNF as increasing $\mathrm{N}$ availability is shown to generally downregulate FLNF (Reed et al. 2011; Smercina et al. 2019a). We predicted that there would be observable rhizosphere metabolite changes in response to $\mathrm{N}$ availability and diazotroph presence. We first hypothesized that total $\mathrm{C}$ exudation by switchgrass would be greater under low $\mathrm{N}$ than high $\mathrm{N}$ conditions as switchgrass attempts to recruit and support diazotrophs and that this response would be amplified by the presence of diazotrophs. Second, we hypothesized that root exudates under low $\mathrm{N}$, particularly when inoculated, would be dominated by $\mathrm{C}$ compounds selective of diazotrophs, such as organic acids (Baldani et al. 2014; Smercina et al. 2019a). Lastly, we hypothesized that differences in root exudate chemistry would be driven by diazotroph presence over $\mathrm{N}$ availability.

\section{MATERIALS AND METHODS}

Experimental setup. Switchgrass root exudates were collected from sterile switchgrass seedlings (cultivar Cave-in-Rock) grown hydroponically in sterile test tubes. To sterilize seeds, $\sim 250$ seeds were placed in three, loosely capped $5 \mathrm{ml}$ tubes inside a desiccator. In a fume hood, seeds were exposed to chlorine gas for $5.5 \mathrm{~h}$. Chlorine gas was generated by adding $3 \mathrm{ml}$ of concentrated hydrochloric acid to $100 \mathrm{ml}$ of $8.25 \%$ sodium hypochlorite. Seedlings were pregerminated from sterile seeds on sterile filter paper in Petri dishes moistened with sterile Milli-Q water (Millipore, Burlington, MA, U.S.A.). Petri dishes of sterile seeds were incubated in the dark at $30^{\circ} \mathrm{C}$ for 5 days. Test tubes were prepared for planting with four Whatman \#114 cellulose filter papers (GE Healthcare Life Sciences, Chicago, IL, U.S.A.) rolled into a ring inside the test tube such that roots would always grow between two layers of filter paper. Filter paper and test tubes were washed three times with high-performance liquid chromatography-grade methanol prior to planting to remove potential contaminants and then autoclaved. Working inside a sterile biosafety cabinet with flame-sterilized forceps, one sterile switchgrass seedling was transplanted between two layers of filter paper in each test tube. After transplanting, $15 \mathrm{ml}$ of a 1/4-strength Hoagland's nutrient solution (1.25 mM KCl, $1.25 \mathrm{mM} \mathrm{CaCl}, 0.25 \mathrm{mM} \mathrm{KH}_{2} \mathrm{PO}_{4}$, $0.5 \mathrm{mM} \mathrm{MgSO} 4,0.012 \mathrm{mM} \mathrm{H} \mathrm{BO}_{3}, 0.002 \mathrm{mM} \mathrm{MnCl} 2 \times 4 \mathrm{H}_{2} \mathrm{O}$, $0.051 \mu \mathrm{M} \mathrm{CuSO}_{4} \times 5 \mathrm{H}_{2} \mathrm{O}, 0.191 \mu \mathrm{M} \mathrm{ZnSO}_{4} \times 7 \mathrm{H}_{2} \mathrm{O}, 0.124 \mu \mathrm{M}$ $\mathrm{Na}_{2} \mathrm{MoO}_{4} \times 2 \mathrm{H}_{2} \mathrm{O}$, and $2.7 \mu \mathrm{M}$ NaFeEDTA) with either high or low $\mathrm{N}$ as $\mathrm{NH}_{4} \mathrm{NO}_{3}$ (described below) added to the bottom of each tube. This facilitated wicking of nutrient solution up from the bottom, allowing roots to grow freely without being submerged in liquid. This also allowed diffusion of root exudates into the nutrient solution for later collection. After planting, each tube was immediately sealed with a gas permeable membrane (BreatheEASIER membrane; Diversified Biotech, Dedham, MA, U.S.A.) to allow gas exchange while enclosing the entire plant in a sterile environment.

Nitrogen was added as ammonium nitrate at either a high $\mathrm{N}$ concentration $\left(3.75 \mathrm{mM} \mathrm{N}-\mathrm{NH}_{4} \mathrm{NO}_{3}\right.$ ) or low $\mathrm{N}$ concentration $\left(0.25 \mathrm{mM} \mathrm{N}-\mathrm{NH}_{4} \mathrm{NO}_{3}\right)$ with a total of 18 tubes per $\mathrm{N}$ treatment. These $\mathrm{N}$ concentrations were chosen based on preliminary experiments that demonstrated switchgrass $\mathrm{N}$-deficiency at the low $\mathrm{N}$ and adequate $\mathrm{N}$ availability at the high $\mathrm{N}$ level (M. L. Friesen, unpublished data). An additional 18 tubes of each $\mathrm{N}$ treatment were prepared as described without planting to be used as background controls. All tubes were placed in a growth chamber on 16-h day cycles ( $155 \mu \mathrm{mol} \mathrm{m} \mathrm{m}^{-2} \mathrm{~s}^{-1}$ light intensity) maintained at $60 \% \mathrm{hu}-$ midity with $23^{\circ} \mathrm{C}$ daytime and $21^{\circ} \mathrm{C}$ nighttime temperatures. After 3 weeks of growth, half of all tubes (both $\mathrm{N}$ treatments and with/ without plants) were inoculated by adding $1 \mathrm{ml}$ of $10^{7} \mathrm{CFU} \mathrm{ml} \mathrm{m}^{-1}$ A. vinelandii DJ (source American Type Culture Collection, ATCC BAA-1303) suspended in 1/4 Hoagland's solution (described above) to each tube. A. vinelandii DJ inoculum was cultured in lysogeny broth at $24^{\circ} \mathrm{C}$ for $\sim 16 \mathrm{~h}$ until optical density $\left(\mathrm{OD}_{600}\right)$ reached 0.1 , corresponding to $10^{7} \mathrm{CFU} \mathrm{ml}^{-1}$ in exponential phase as verified in preliminary work. A. vinelandii DJ cultures were then centrifuged at 3,220 $\times \mathrm{g}$ for $5 \mathrm{~min}$, supernatant was discarded, and then cultures were resuspended in the same volume of sterile $1 / 4$ Hoagland's solution prior to plant inoculation. Uninoculated seedlings received $1 \mathrm{ml}$ of sterile 1/4 Hoagland's solution in place of inoculation. Plants were then grown an additional 3 days, with and without $A$. vinelandii DJ. This resulted in four treatment groups (high $\mathrm{N}$, high $\mathrm{N}+A$. vinelandii $\mathrm{DJ}$, low $\mathrm{N}$, and low $\mathrm{N}+A$. vinelandii DJ) with and without plants for a total of 72 tubes (nine tubes per treatment). Sterility of all tubes was confirmed prior to inoculation by plating nutrient solution from each sample onto tryptone yeast extract agar plates $\left(5 \mu \mathrm{l}\right.$ each). Plates were placed in a $30^{\circ} \mathrm{C}$ incubator for 3 days and then examined for growth.

Sample harvest. Plant biomass and metabolite samples were harvested 3 days after inoculation. All harvest work was done in a sterile laminar flow hood with flame-sterilized equipment. The porous membrane of each tube was removed and then the filter paper containing a seedling was carefully removed from the tube. Shoot biomass was clipped and root systems were carefully excised from the filter paper. Shoot and root biomass were dried at $60^{\circ} \mathrm{C}$ for at least 3 days to determine above- and belowground biomass, respectively. All nutrient solution from the bottom of each tube was transferred to $50 \mathrm{ml}$ conical tubes. The filter paper was placed on three sterile pipette tips inside the same $50 \mathrm{ml}$ tube and then the tube was centrifuged at $1,610 \times g$ for 5 min to collect residual solution from the filter paper. The solution was then transferred to a $15 \mathrm{ml}$ conical tube and centrifuged at $3,220 \times g$ to pellet debris. Finally, the supernatant from three replicates per treatment group was collected and pooled for analysis, resulting in a total of 24 samples for nuclear magnetic resonance (NMR) spectroscopy analysis, including 12 background control samples. Each of these samples represents metabolites produced during the entire course of the experiment ( 3 weeks of plant growth +3 days of inoculation) and 
may include cellular debris as no final filtration step was performed before NMR analysis. Due to microbial contamination in one tube, only two samples were pooled for low $\mathrm{N}$ seedling treatment replicate three. Samples were frozen at $-80^{\circ} \mathrm{C}$ until lyophilization.

NMR analysis. Lyophilized material from each tube was weighed and resuspended in $180 \mu \mathrm{l}$ of $0.5 \mathrm{mM}$ dDSS (4,4-dimethyl4-silapentane-1-sulfonic acid; CAS-no. 2039-96-5) in $10 \% \mathrm{D}_{2} \mathrm{O} /$ $90 \% \mathrm{H}_{2} \mathrm{O}$ for NMR analysis. NMR spectra for all samples were collected using a Varian Direct Drive 600-MHz NMR spectrometer with a 5-mm triple resonance cold probe. NMR spectra were collected using standard Chenomx data guidelines (Weljie et al. 2006) employing a $1 \mathrm{D}{ }^{1} \mathrm{H}$ NOESY presaturation experiment and were processed, assigned, and analyzed using Chenomx NMR Suite 8.3 (Edmonton, AB, Canada). Spectra were quantified based on intensity relative to the $0.5 \mathrm{mM}$ DSS-d6 (3-(Trimethylsilyl)-1propanesulfonic acid-d6 sodium salt; CAS-no. 284664-85-3) internal standard. Sample metabolites were determined by matching chemical shift, J-coupling, and intensity against NMR signals of metabolites in the Chenomx library. Intensities of metabolite peaks are calibrated against final concentrations of the DSS-d6 internal standard for all metabolites in the Chenomx library, thus allowing us to determine metabolite concentrations. Raw metabolite concentrations were reported as micromolar concentrations. It is important to note that not all compounds within the lyophilized material are detected via NMR and that a lack of detection does not mean an absence of the compound, but merely that the concentrations are below the NMR detection limit. Further, in this study we are specifically focused on $\mathrm{C}$ containing compounds.

Data analysis and statistics. Raw metabolite concentrations were converted from micromoles to nanomoles per milligrams of dry material by first multiplying by the volume of dDSS used in resuspension (i.e., $1.8 \times 10^{-4}$ liter) to determine micromoles of metabolite, then converting to nanomoles by multiplying this by 1,000 , and finally dividing by milligrams of dry, lyophilized material. Final compound concentrations, as nanoles of detected metabolite per gram of dry material, were calculated by subtracting average compound concentration in background controls from samples under each $\mathrm{N}$ and inoculation treatment. Relative abundance was calculated as the nanomoles of detected metabolite per gram of dry material divided by total nanomoles of all detected metabolites per gram of dry material, thus relative abundance is nanomoles of detected metabolite per nanomoles of total metabolites.

The distributions of metabolite concentrations were nonnormal, requiring nonparametric statistical procedures. Therefore, comparisons of concentration and relative abundance of specific compounds by $\mathrm{N}$ treatment or inoculation were carried out using Friedman's test, nonparametric two-way analysis of variance with Tukey's adjustment for multiple comparisons in SAS (SAS v. 9.1). Principal coordinates analysis ( $\mathrm{PCoA}$ ) was conducted in $\mathrm{R}$ using the vegan package tools vegdist and adonis ( $\mathrm{R}$ version 3.6.0; Oksanen et al. 2010) with Bray-Curtis dissimilarity. Correlations of the dissimilarity matrix and compound group concentrations were carried out using the vegan tool envfit. We calculated 95\% confidence ellipses for PCoA using dataEllipse in the car package ( $\mathrm{R}$ version 3.6.0; Fox and Weisberg 2018).

\section{RESULTS}

Metabolite concentrations. We successfully collected and analyzed the metabolite profiles of switchgrass and switchgrass with A. vinelandii DJ grown in a sterile, semihydroponic system. We detected and identified 34 rhizosphere metabolites representing eight compound groups including alcohols/polyols, amines/amides, carbohydrates, cholines, ketones, nucleic acids, organic acids, and quinones (Table 1). Total measured rhizosphere metabolite concentration (nanomole detected metabolite per gram of dry material) was on average $2 \times$ greater for high $\mathrm{N}$ plants than low $\mathrm{N}$ plants $(\mathrm{F}=$ $10.89, P=0.0109$; Fig. 1A). Though not significant, there was a trend toward greater detected metabolite concentrations with inoculation ( $\mathrm{F}=3.56, P=0.0961$; Fig. 1B). There was no significant interaction effect of $\mathrm{N}$ treatment and inoculation on total measured rhizosphere metabolite concentration.

We explored differences in concentrations of specific compounds between $\mathrm{N}$ treatments and inoculation treatments (Supplementary Table S1). Arabinose, fructose, glucose, glycine, lactate, and methanol were all significantly greater under high $\mathrm{N}$ and were not impacted by inoculation (Supplementary Table S2). In contrast, lysine and uracil were significantly greater when plants were inoculated $(P=0.0043$ and $P<0.0001$, respectively) and were not impacted by $\mathrm{N}$ availability. Some compounds also had interactions between $\mathrm{N}$ treatment and inoculation, including allantoin, betaine, and valine (Fig. 2; Supplementary Table S2). Allantoin was greatest when plants were under high $\mathrm{N}$ and inoculated with no significant differences between high N, low N, or low N with inoculation (Fig. $2 \mathrm{~A}, P<0.0001)$. Betaine was also greatest in the high $\mathrm{N}$ with inoculation treatment followed by low $\mathrm{N}$ with inoculation, low $\mathrm{N}$, and finally high $\mathrm{N}$ (Fig. 2B, $P=0.0123$ ). Valine was greatest under low $\mathrm{N}$ without inoculation and high $\mathrm{N}$ with inoculation with no significant differences between high $\mathrm{N}$ and low $\mathrm{N}$ with inoculation (Fig. 2C, $P<0.0001$ ).

We also explored differences in concentration of compound classes across $\mathrm{N}$ and inoculation treatments (Supplementary Table S3). Total concentration (nanomole per gram of dried material) of carbohydrates were significantly greater under high $\mathrm{N}(P=0.0009)$, while total choline concentrations were significantly greater under low N $(P=0.0055)$. Total amines/amides and nucleic acid concentrations were greater when plants were inoculated with A. vinelandii DJ than when uninoculated $(P=0.0384$ and 0.0038 , respectively).

Relative abundance. Because of differences in total metabolite concentration between $\mathrm{N}$ treatments, we also compared relative abundance of metabolite compound groups between $\mathrm{N}$ treatments (Fig. 3; Supplementary Table S3). High N samples were dominated by carbohydrates with $55.1 \%$ of detected C compounds belonging to this compound group. Carbohydrates only represented $8.1 \%$ of detected C compounds in low $\mathrm{N}$ samples. We found organic acids to be the most abundant group in low $\mathrm{N}$ samples, representing over $28 \%$ of detected $\mathrm{C}$ compounds in low $\mathrm{N}$ samples. Overall, high $\mathrm{N}$ samples had a greater abundance of carbohydrates $(P=0.0001)$ compared with low $\mathrm{N}$ samples. Low $\mathrm{N}$ samples had a greater abundance of cholines $(P=0.0008)$ and organic acids $(P=$ $0.0010)$ compared with high $\mathrm{N}$ samples. Carbon compounds detected in low $\mathrm{N}$ samples were more evenly distributed across compound groups with few differences between compound groups. Relative abundances of N-rich compound classes, including amines/amides and nucleic acids, were not significantly impacted by $\mathrm{N}$ availability, but were altered with inoculation. Relative abundance of nucleic acids $(P=0.0144)$ was found to be significantly greater in inoculated rhizospheres. Similarly, amines/amides relative abundance showed a marginal response $(P=0.0667)$, trending toward greater abundance with inoculation. Relative abundance of carbohydrates $(P=0.0382)$ was also significantly impacted by inoculation and was greater in uninoculated rhizospheres.

Rhizosphere metabolic fingerprint. We also examined the rhizosphere metabolite "fingerprints" based on relative abundance of all identified compounds in each sample (Table 1). 
PCoA shows fingerprints were driven by $\mathrm{N}$ treatment $\left(R^{2}=\right.$ 0.31096, $P=0.0004$; Fig. 4) and inoculation $\left(R^{2}=0.16805, P=\right.$ $0.0199)$, with no significant interaction. Environmental factor analysis indicates that spatial distribution in the PCoA is significantly correlated with carbohydrate $\left(R^{2}=0.7142 ; P=0.005\right)$ and choline $\left(R^{2}=0.5931, P=0.029\right)$ concentrations and marginally correlated with organic acid concentrations $\left(R^{2}=0.4184\right.$, $P=0.081)$.

Plant biomass. We found differences in shoot biomass by $\mathrm{N}$ treatment $(P=0.00503$; Fig. $5 \mathrm{~A})$, with over $2.5 \times$ greater shoot biomass on average under high $\mathrm{N}$ than under low $\mathrm{N}$. Though we found no statistically significant difference in root biomass between high and low $\mathrm{N}(P=0.259$; Fig. $5 \mathrm{C})$, there was a trend toward greater root biomass under high $\mathrm{N}$ versus low $\mathrm{N}$ conditions. There were no differences in shoot or root biomass by inoculation treatment (Fig. 5B and D, respectively).

\section{DISCUSSION}

Our results indicate that $\mathrm{N}$ availability may be a stronger driver of rhizosphere metabolite chemistry than diazotroph presence. $\mathrm{N}$ availability was the main driver of total root $\mathrm{C}$ exudation with high $\mathrm{N}$ plants exuding twofold greater total detected $\mathrm{C}$ compounds. We also found high $\mathrm{N}$ availability resulted in $2.5 \times$ greater shoot biomass while root biomass was not significantly different between $\mathrm{N}$ treatments, suggesting that

TABLE 1

Relative abundances (nmol detected metabolite $\mathrm{nmol}^{-1}$ of total metabolites) of all compounds by treatment groups ${ }^{\mathrm{a}}$

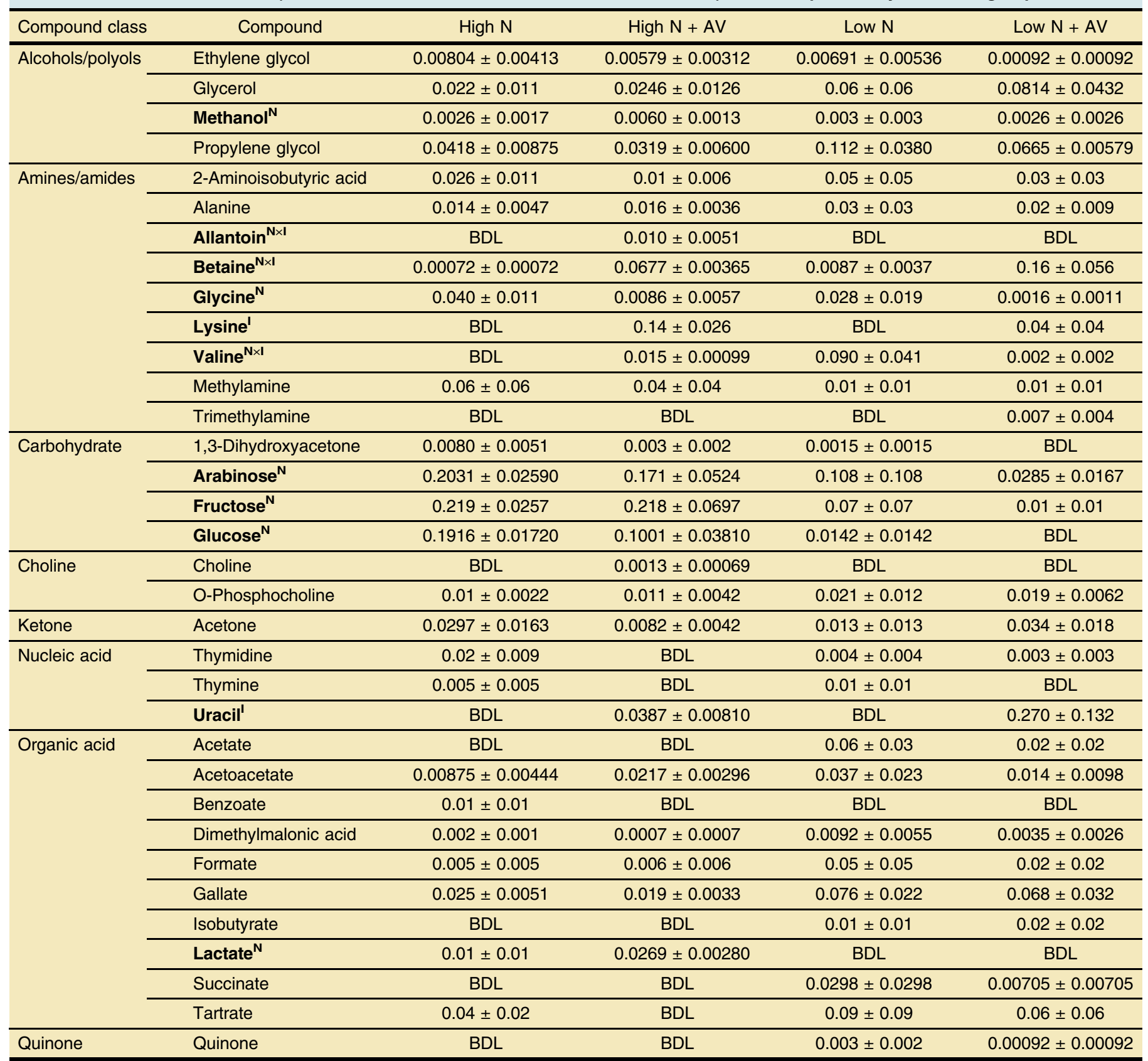

${ }^{a} \mathrm{BDL}=$ below detection limit. Compounds with significant treatment differences appear in bold. $\mathrm{AV}=$ Azotobacter vinelandii DJ. ${ }^{\mathrm{N}}$ Indicates significant $\mathrm{N}$ treatment effect. ' Indicates significant inoculum treatment effect. ${ }^{\mathrm{N} \times l}$ Indicates significant interaction effect. 
increased exudation may be the result of greater photosynthetic activity and aboveground productivity rather than increased root biomass. We found no differences in shoot or root biomass with inoculation treatment; however, this may indicate that roots were not exposed to inoculation long enough to see a large response in growth.
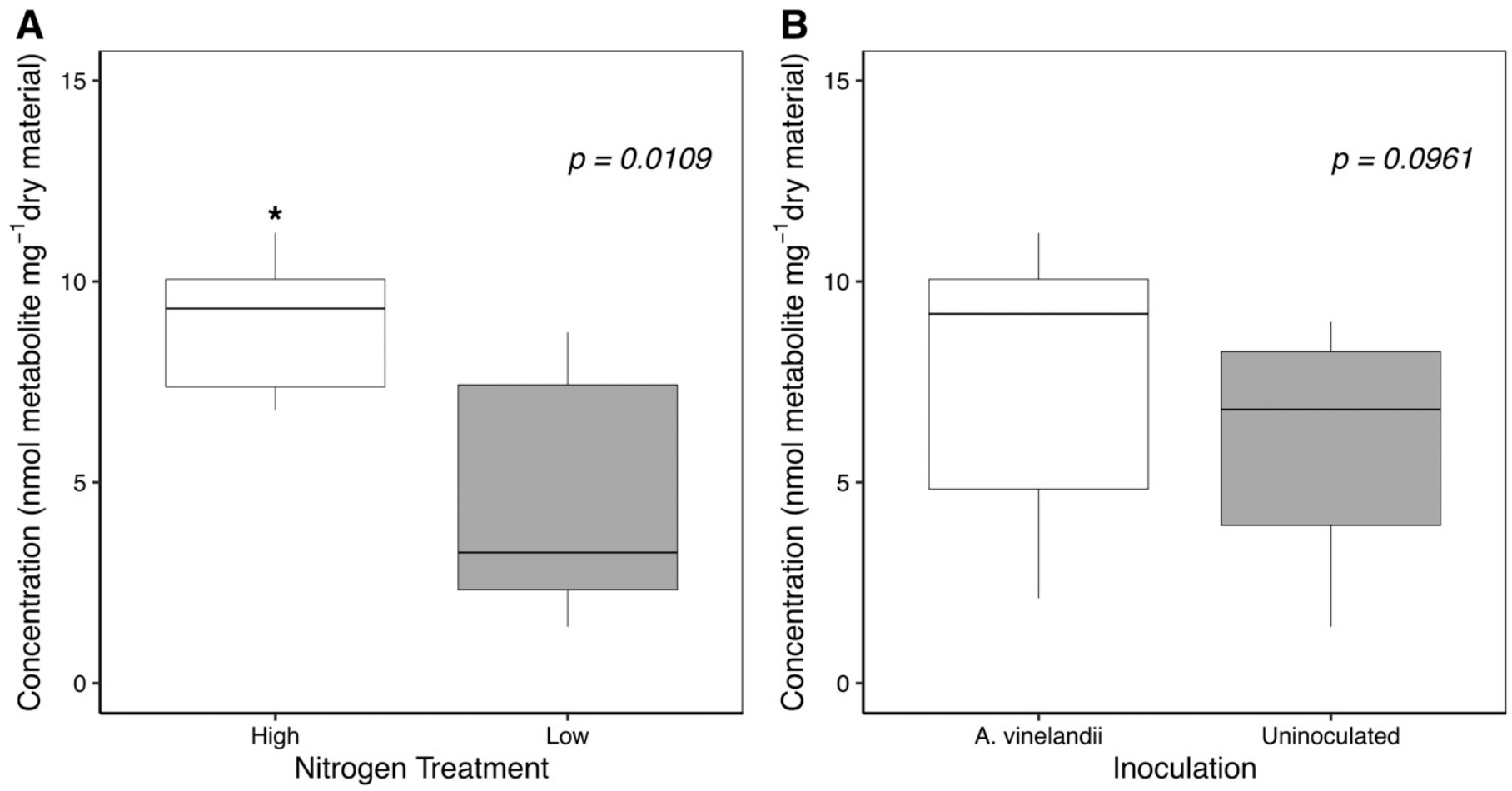

Fig. 1. Concentration of detected total metabolite carbon by $\mathbf{A}$, nitrogen treatment and $\mathbf{B}$, inoculation treatment. Boxplots show full range of concentrations with the solid black line denoting average concentration $(n=6)$. Asterisks indicate significant differences for $P<0.05$. Differences in total metabolite carbon were not significant by inoculation treatment.

A

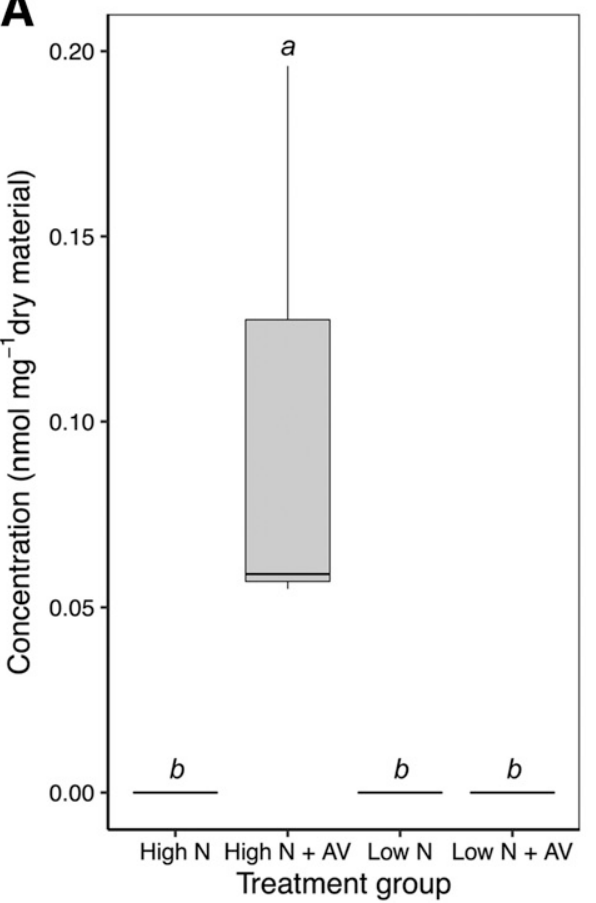

B

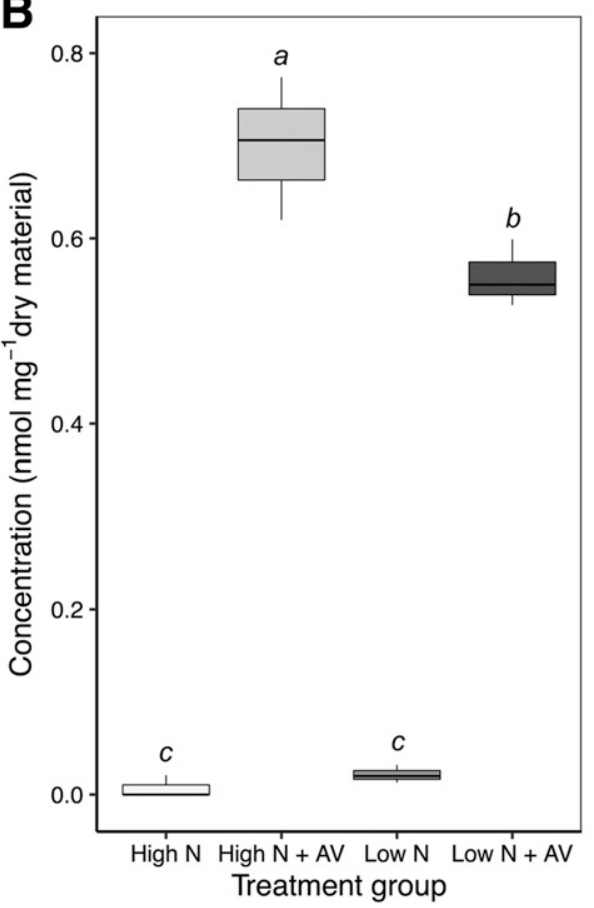

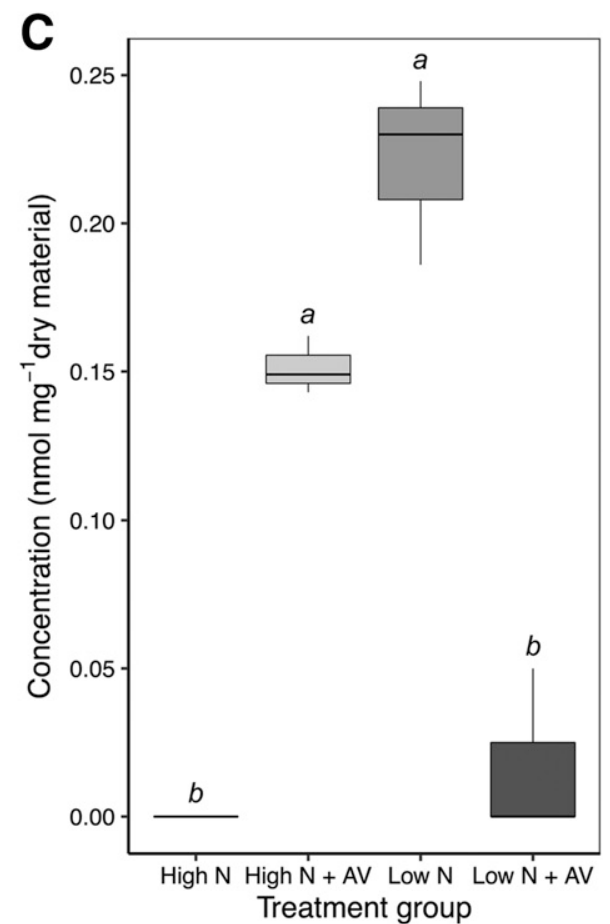

Fig. 2. Concentration of A, allantoin, B, betaine, and C, valine compounds that had significant nitrogen by inoculation treatment effects. Boxplots show full range of concentrations with the solid black line denoting average concentration $(n=3)$. Lowercase letters indicate significant differences for nitrogen by inoculation interactions where $P<0.05$. AV $=$ Azotobacter vinelandii DJ. 
Total metabolite concentration also did not show a response to inoculation. This was surprising as we hypothesized that switchgrass would increase $\mathrm{C}$ exudation under low $\mathrm{N}$ in order to recruit and/or support diazotrophs. Though, there are of course many other drivers of exudation besides recruitment of diazotrophs, including biotic and abiotic stresses (Bertin et al. 2003). We also hypothesized that inoculation would further increase the observed differences in exudation by $\mathrm{N}$ treatment, but found no such result for total exuded C compounds. It is important to note that plants in our study had a short exposure to diazotrophs (i.e., 3 days) and we were unable to directly assess inoculum survival in association with the plants. Together, these may have limited any detectable response in $\mathrm{C}$ quantity to diazotroph presence. However, a separate experiment to evaluate inoculum survival under conditions similar to the original experiment (note: plants were not included; carbohydrates and organic acids were supplied as the $\mathrm{C}$ source-see Supplementary Materials) revealed potential for inoculum survival with an average inoculum concentration of $\sim 1.6 \times 10^{7} \mathrm{CFU} \mathrm{ml}{ }^{-1}$ (Supplementary Fig. S1) and an average survival rate of $\sim 33 \%$ (Supplementary Fig. S2). Therefore, it is plausible that there was inoculum survival in the

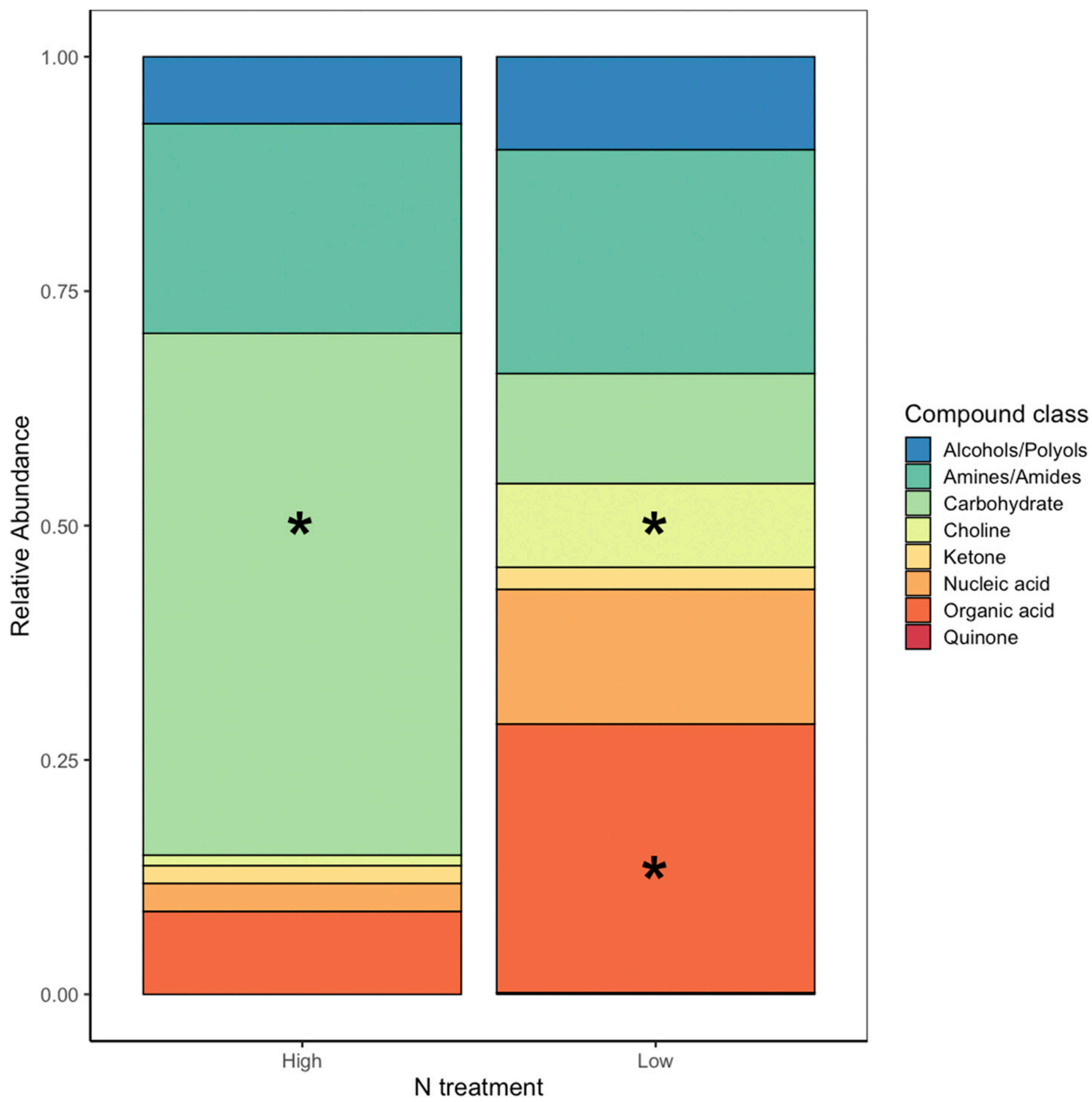

Fig. 3. Relative abundance of compound groups by nitrogen treatment ( $n=6$ per nitrogen treatment). High N rhizospheres were carbohydrate dominated (55.1\%), while low $\mathrm{N}$ rhizospheres were organic acid dominated (28.7\%). Asterisks indicate significant difference between relative abundance at high versus low nitrogen where $P<0.05$. Asterisks are placed on the bar with greater relative abundance. 
original experiment. However, total quantity of exuded $\mathrm{C}$ may not be as important to diazotroph activity as $\mathrm{C}$ chemistry (Smercina et al. 2019a) and indeed in this study, we find that changes in metabolite chemistry provide more insight into the switchgrassdiazotroph interaction than total exuded $\mathrm{C}$.

Consistent with our second hypothesis, we found that low $\mathrm{N}$ rhizospheres had a greater relative abundance of organic acids compared with high $\mathrm{N}$ rhizospheres. Organic acids may be a preferred $\mathrm{C}$ source of many diazotrophs and are often used to isolate diazotrophs from soils (Baldani et al. 2014). The greater relative abundance of organic acids observed under low $\mathrm{N}$ could indicate the ability of switchgrass to support diazotrophs through increased exudation of preferable $\mathrm{C}$ sources. However, we found no significant differences in organic acid concentration or relative abundance with inoculation or $\mathrm{N} \times$ inoculation effects, indicating that these changes may not be directly in response to diazotroph presence. We also found high $\mathrm{N}$ rhizospheres were dominated by carbohydrates, representing over $55 \%$ of detected metabolites versus just over $8 \%$ of detected metabolites in low $\mathrm{N}$ rhizospheres. We did not find any significant differences in carbohydrate concentration by inoculation treatment or $\mathrm{N} \times$ inoculation effects, but found uninoculated rhizospheres to have a greater relative abundance of carbohydrates than inoculated rhizospheres. These observed differences in carbohydrate concentrations by $\mathrm{N}$ treatment most likely resulted from greater photosynthetic activity in the high $\mathrm{N}$ plants, which had greater aboveground biomass, rather than a response to diazotroph presence. Interestingly, the differences in carbohydrate relative abundance by inoculation treatment may indicate that $A$. vinelandii DJ was actively consuming exuded carbohydrates (Supplementary Fig. S3). Although there is evidence that organic acids are a preferable $\mathrm{C}$ source for some diazotrophs

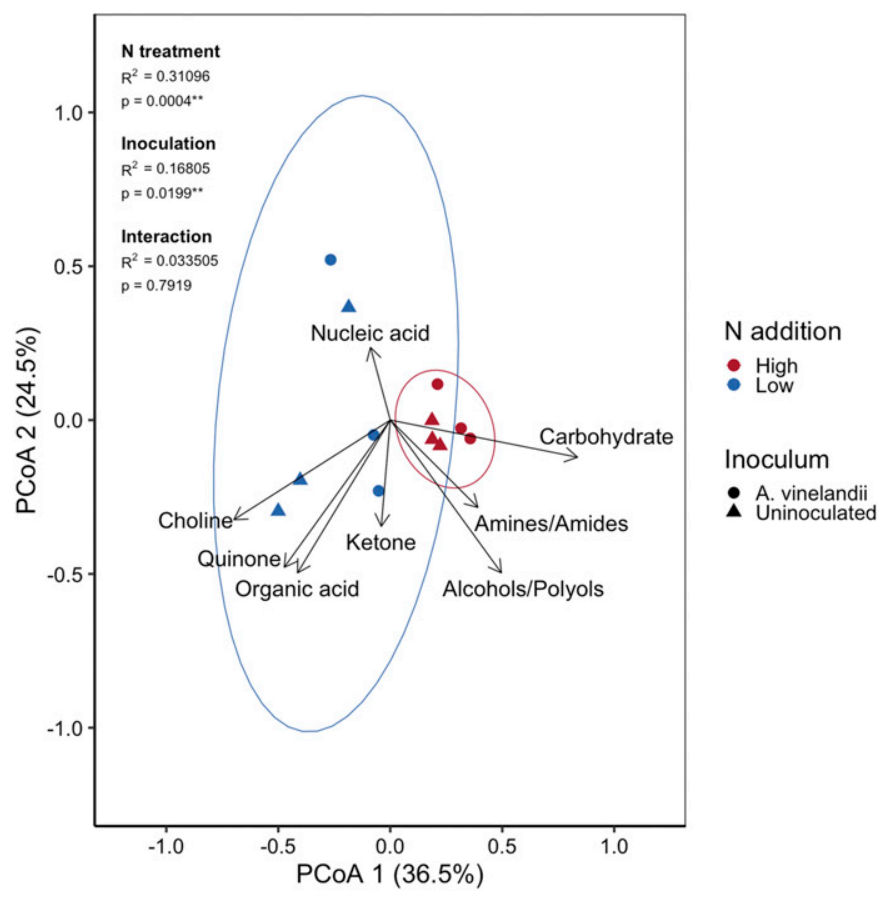

Fig. 4. Principal coordinates analysis displaying Bray-Curtis dissimilarity based on relative abundance for all detected compounds. Ellipses represent $95 \%$ confidence by nitrogen treatments; $95 \%$ confidence ellipses for inoculation are not shown. Each point is represented by one sample. Overlaid vectors display correlations of the Bray-Curtis dissimilarity matrix with concentrations of each detected compound class.
(Baldani et al. 2014), we did not find evidence of organic acid uptake by $A$. vinelandii DJ (i.e., no significant difference in organic acid relative abundance by inoculation treatment). Further, we found no significant differences in $A$. vinelandii DJ survival when organic acids versus carbohydrates were provided as a $\mathrm{C}$ source (Supplementary Figs. S1 and S2).

Using the relative abundance of all compounds, we generated rhizosphere metabolite profiles, or "fingerprints," to directly compare overall differences in metabolite chemistry across treatments. We found that fingerprints differed significantly between $\mathrm{N}$ and inoculation treatments, but found no significant interaction effect indicating that switchgrass response to diazotroph presence was not influenced by $\mathrm{N}$ availability. We also found that overall metabolite profiles are correlated with carbohydrate and choline concentrations and marginally with organic acid concentrations. Collectively, these results tell us that $\mathrm{N}$ availability may influence the ability of switchgrass to support FLNF (i.e., C availability as carbohydrates versus organic acids). Though organic acid concentrations were not significantly different between high and low $\mathrm{N}$, low $\mathrm{N}$ rhizospheres trended toward greater concentrations of organic acids, while carbohydrates were significantly greater under high N (Supplementary Fig. S4). These shifts in C source and availability may influence the potential for FLNF to occur in the switchgrass rhizosphere, especially if diazotrophs rely on root exuded to support $\mathrm{N}$-fixation as hypothesized.

Concentrations of most compounds in our study showed no significant response to inoculation treatment, thus limiting evidence for direct switchgrass-diazotroph interaction. However, we did find responses in both uracil and lysine concentrations, two compounds potentially important to plant-microbe interactions. Uracil could simply be of microbial origin; previous work shows that uracil is actively excreted from N-fixing bacteria (Azotobacter agilis B 170; Katayama et al. 1967), but it is also known to be important in the production of small, noncoding RNAs involved in plant-microbe interactions (Weiberg et al. 2014). Similarly, lysine is a precursor to glutamate, an important signaling compound for plant growth and for plant response to environmental conditions, particularly stress responses like osmotic-stress (Arruda et al. 2000; Galili 2002). Lysine is also produced in large amounts by A. vinelandii strains (Gonzalez-Lopez et al. 1983) and has been shown to be essential for ATP binding to the iron cofactor of nitrogenase synthesized by A. vinelandii (Seefeldt et al. 1992). It is plausible that the lysine detected here was produced by the $A$. vinelandii DJ inoculum and may indicate active $\mathrm{N}$-fixation by $A$. vinelandii DJ. Further, this production of lysine by $A$. vinelandii DJ may have induced stress responses in switchgrass, which is further evidenced by detection of compounds like allantoin, betaine, and valine.

Allantoin, betaine, and valine have all been associated with plant stress response, particularly osmotic stress response. All three compounds were detected in this study and were found to have significant $\mathrm{N} \times$ inoculation responses. Allantoin concentration under high $\mathrm{N}+A$. vinelandii $\mathrm{DJ}$ was over twofold greater than the other three treatment combinations. Allantoin has been shown to play a role in osmotic stress response of Arabidopsis (Watanabe et al. 2014). It is plausible that allantoin production was induced because of lysine production by $A$. vinelandii DJ or because of the hydroponic growth system. Allantoin presence could also be indicative of phosphorus deficiency (Tawaraya et al. 2014). This is plausible in our system, particularly in the high $\mathrm{N}+A$. vinelandii $\mathrm{DJ}$ treatment, as competition for nutrients between plants and microbes may result in $\mathrm{P}$ limitation relative to $\mathrm{N}$ causing stoichiometric imbalances (Sterner and Elser 2002). Allantoin may also be indicative of active $\mathrm{N}$ recycling from microbial biomass as it is also an intermediate product of purine catabolism (Watanabe et al. 
2014). Greater biomass (both plant and potentially microbial) in the high $\mathrm{N}+A$. vinelandii $\mathrm{DJ}$ treatment would result in greater availability of biomass-derived $\mathrm{N}$ and therefore, we could expect greater allantoin production through the purine catabolism pathway.

Betaine was also detected in the rhizosphere and may indicate plant and microbial stress response (Sakamoto and Murata 2002; Wargo 2013). In plants, betaine production can occur in response to high salt environments (Sakamoto and Murata 2002), while in bacteria, betaine is an important osmoprotectant (Borton et al. 2018; Wood 2011). Betaine concentrations were generally greater with inoculation, but showed inverse effects of $\mathrm{N}$ treatment where betaine concentration was greater in high $\mathrm{N}$ when inoculated, but greater in low $\mathrm{N}$ when uninoculated. This suggests that betaine was produced by both plants and microbes in our system. While some microbes convert betaine to glycine which would provide a pathway for $\mathrm{N}$ cycling (Wargo 2013), it seems most likely that betaine production was a stress response as concentrations were generally greater where we might expect greater plant and microbial biomass.

Lastly, valine was detected in our system and showed complex treatment responses which may indicate multiple drivers of valine production. When uninoculated, valine production most likely
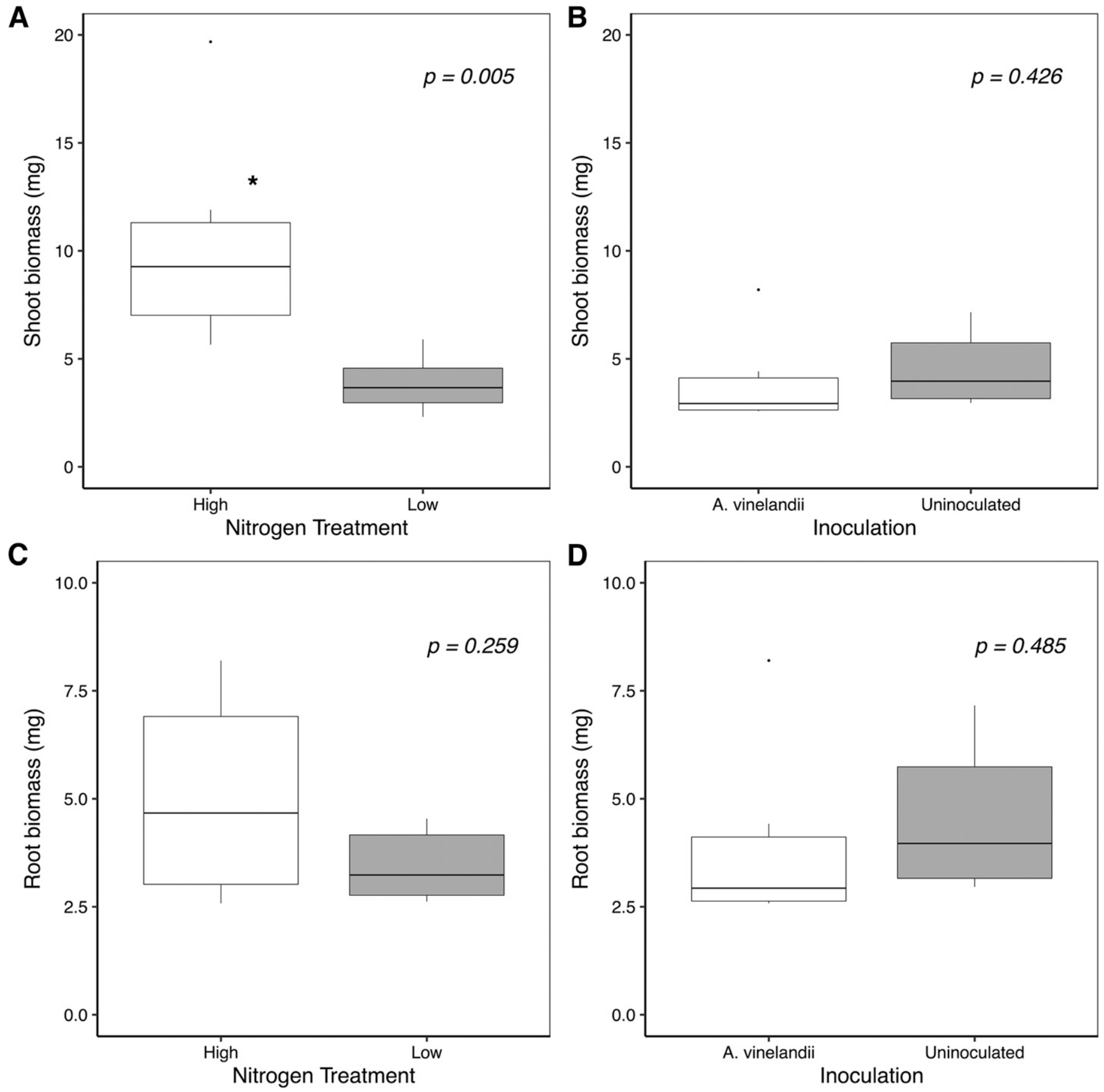

Fig. 5. Shoot and root biomass by $\mathbf{A}$ and $\mathbf{C}$, nitrogen treatment and $\mathbf{B}$ and $\mathbf{D}$, inoculation treatment. Boxplots show full range of concentrations with the solid black line denoting average concentration $(n=6)$. Asterisk indicates significantly greater biomass by nitrogen treatment. Differences in shoot and root biomass were not significant by inoculation treatment. Root biomass differences were not significant by nitrogen treatment. 
indicates stress response in plants, potentially osmotic stress (Gargallo-Garriga et al. 2018). However, when inoculated, valine may also indicate biofilm formation by the inoculum (Valle et al. 2008). Our pattern of results for valine suggests a combination of the two where uninoculated plants in low $\mathrm{N}$ conditions exhibit strong stress response which is alleviated under high $\mathrm{N}$, and inoculum are forming biofilms under high $\mathrm{N}$ conditions, but not as strongly under low $\mathrm{N}$ conditions. Collectively, the presence of allantoin, betaine, and valine suggest plants may have been responding as if osmotically stressed, though this response was altered by $\mathrm{N}$ availability and inoculation.

In conclusion, our findings indicate that switchgrass rhizosphere metabolite chemistry is driven by $\mathrm{N}$ availability and may respond to the presence of a diazotroph inoculum. Plant responses to diazotroph presence may have been dampened by short exposure time and potentially reduced inoculum survival. Overall greater detected $\mathrm{C}$ compounds under high $\mathrm{N}$ was counter to our hypothesis that switchgrass would exude more $\mathrm{C}$ when $\mathrm{N}$-limited and indicates that aboveground productivity rather than plant recruitment of diazotrophs is a strong driver of exudation. Metabolite chemistry was also driven more strongly by $\mathrm{N}$ availability with a greater relative abundance and concentration of carbohydrates under high $\mathrm{N}$ and greater relative abundance of organic acids under low $\mathrm{N}$. These changes in chemistry suggest $\mathrm{N}$ availability may influence the switchgrass-diazotroph interaction impacting the potential for switchgrass to benefit from FLNF-derived N.

\section{ACKNOWLEDGMENTS}

We thank the editor and all reviewers for constructive feedback that helped to improve this manuscript.

\section{LITERATURE CITED}

Arruda, P., Kemper, E. L., Papes, F., and Leite, A. 2000. Regulation of lysine catabolism in higher plants. Trends Plant Sci. 5:324-330.

Bais, H. P., Weir, T. L., Perry, L. G., Gilroy, S., and Vivanco, J. M. 2006. The role of root exudates in rhizosphere interactions with plants and other organisms. Annu. Rev. Plant Biol. 57:233-266.

Bahulikar, R. A., Torres-Jerez, I., Worley, E., Craven, K., and Udvardi, M. K. 2014. Diversity of nitrogen-fixing bacteria associated with switchgrass in the native tallgrass prairie of Northern Oklahoma. Appl. Environ. Microbiol. 80: 5636-5643.

Baldani, J. I., Reis, V. M., Videira, S. S., Boddey, L. H., and Baldani, V. L. D. 2014. The art of isolating nitrogen-fixing bacteria from non-leguminous plants using Nfree semi-solid media: A practical guide for microbiologists. Plant Soil 384:413-431.

Bertin, C., Yang, X., and Weston, L. A. 2003. The role of root exudates and allelochemicals in the rhizosphere. Plant Soil 256:67-83.

Borton, M. A., Hoyt, D. W., Roux, S., Daly, R. A., Welch, S. A., Nicora, C. D., et al. 2018. Coupled laboratory and field investigations resolve microbial interactions that underpin persistence in hydraulically fractured shales. Proc. Natl. Acad. Sci. 115:E6585-E6594.

Fox, J., and Weisberg, S. 2018. An R Companion to Applied Regression. Sage Publications, Thousand Oaks, CA.

Galili, G. 2002. New insights into the regulation and functional significance of lysine metabolism in plants. Annu. Rev. Plant Biol. 53:27-43.

Gargallo-Garriga, A., Preece, C., Sardans, J., Oravec, M., Urban, O., and Peñuelas, J. 2018. Root exudate metabolomes change under drought and show limited capacity for recovery. Sci. Rep. 8:12696.

Gonzalez-Lopez, J., Salmeron, V., Moreno, J., and Ramos-Cormenzana, A. 1983. Amino acids and vitamins produced by Azotobacter vinelandii ATCC 12837 in chemically-defined media and dialysed soil media. Soil Biol. Biochem. 15:711-713.
Jones, D. L., Nguyen, C., and Finlay, R. D. 2009. Carbon flow in the rhizosphere: Carbon trading at the soil-root interface. Plant Soil 321: 5-33.

Katayama, T., Kobayashi, M., and Okuda, A. 1967. Nucleic acids in the culture fluids of Rhodopseudomonas capsulatus and other heterotrophic bacteria. Soil Sci. Plant Nutr. 13:101-106.

Oksanen, J., Blanchet, F. G., Kindt, R., Legendre, P., O'Hara, R. B., Simpson, G. L., Solymos, P., Stevens, M. H. H., and Wagner, H. 2010. Vegan: Community ecology package. R package version 1.17-4. https://cran.r-project.org/web/ packages/vegan/

Parrish, D. J., and Fike, J. H. 2005. The biology and agronomy of switchgrass for biofuels. BPTS 24:423-459.

Reed, S. C., Cleveland, C. C., and Townsend, A. R. 2011. Functional ecology of free-living nitrogen fixation: A contemporary perspective. Annu. Rev. Ecol. Evol. Syst. 42:489-512.

Robertson, G. P., Hamilton, S. K., Barham, B. L., Dale, B. E., Izaurralde, R. C., Jackson, R. D., Landis, D. A., Swinton, S. M., Thelen, K. D., and Tiedje, J. M. 2017. Cellulosic biofuel contributions to a sustainable energy future: Choices and outcomes. Science 356:eaal2324.

Roley, S. S., Duncan, D. S., Liang, D., Garoutte, A., Jackson, R. D., Tiedje, J. M., and Robertson, G. P. 2018. Associative nitrogen fixation (ANF) in switchgrass (Panicum virgatum) across a nitrogen input gradient. PLoS One 13:e0197320.

Roley, S. S., Xue, C., Hamilton, S. K., Tiedje, J. M., and Robertson, G. P. 2019. Isotopic evidence for episodic nitrogen fixation in switchgrass (Panicum virgatum L.). Soil Biol. Biochem. 129:90-98.

Ruan, L., Bhardwaj, A. K., Hamilton, S. K., and Robertson, G. P. 2016. Nitrogen fertilization challenges the climate benefit of cellulosic biofuels. Environ. Res. Lett. 11:064007.

Sakamoto, A., and Murata, N. 2002. The role of glycine betaine in the protection of plants from stress: Clues from transgenic plants. Plant Cell Environ. 25: 163-171.

Sasse, J., Martinoia, E., and Northen, T. 2018. Feed your friends: Do plant exudates shape the root microbiome? Trends Plant Sci. 23:25-41.

Seefeldt, L. C., Morgan, T. V., Dean, D. R., and Mortenson, L. E. 1992. Mapping the site(s) of MgATP and MgADP interaction with the nitrogenase of Azotobacter vinelandii. Lysine 15 of the iron protein plays a major role in MgATP interaction. J. Biol. Chem. 267:6680-6688.

Smercina, D. N., Evans, S. E., Friesen, M. L., and Tiemann, L. K. 2019a. To fix or not to fix: Controls on free-living nitrogen fixation in the rhizosphere. Appl. Environ. Microbiol. 85:e02546-e18.

Smercina, D. N., Evans, S. E., Friesen, M. L., and Tiemann, L. K. 2019b. Optimization of the ${ }^{15} \mathrm{~N}_{2}$ incorporation and acetylene reduction methods for free-living nitrogen-fixation. Plant Soil 445:595-611.

Sterner, R. W., and Elser, J. J. 2002. Ecological Stoichiometry: The Biology of Elements from Molecules to the Biosphere. Princeton University Press, Princeton, NJ.

Tawaraya, K., Horie, R., Saito, S., Wagatsuma, T., Saito, K., and Oikawa, A. 2014. Metabolite profiling of root exudates of common bean under phosphorus deficiency. Metabolites 4:599-611.

Valle, J., Da Re, S., Schmid, S., Skurnik, D., D’Ari, R., and Ghigo, J. M. 2008. The amino acid valine is secreted in continuous-flow bacterial biofilms. J. Bacteriol. 190:264-274.

Wargo, M. J. 2013. Homeostasis and catabolism of choline and glycine betaine: lessons from Pseudomonas aeruginosa. Appl. Environ. Microbiol. 79: 2112-2120.

Watanabe, S., Matsumoto, M., Hakomori, Y., Takagi, H., Shimada, H., and Sakamoto, A. 2014. The purine metabolite allantoin enhances abiotic stress tolerance through synergistic activation of abscisic acid metabolism. Plant Cell Environ. 37:1022-1036.

Weiberg, A., Wang, M., Bellinger, M., and Jin, H. 2014. Small RNAs: A new paradigm in plant-microbe interactions. Annu. Rev. Phytopathol. 52: 495-516.

Weljie, A. M., Newton, J., Mercier, P., Carlson, E., and Slupsky, C. M. 2006. Targeted profiling: Quantitative analysis of H-1 NMR metabolomics data. Anal. Chem. 78:4430-4442.

Wood, J. M. 2011. Bacterial osmoregulation: A paradigm for the study of cellular homeostasis. Annu. Rev. Microbiol. 65:215-238. 